\title{
Peculiarities of the Stellar Population of Bars in the LMC and Other Barred Galaxies
}

\author{
A.S. Gusev
}

Sternberg Astronomical Institute, Universitetsky Pr., 13, 119899, Moscow B-234, Russia

\begin{abstract}
.
The age distributions in the bar and disk of the Large Magellanic Cloud are studied using data for 262 clusters with known ages, of which 89 are located in the bar. The spatial distributions for clusters in different age groups are investigated. Epochs of active star formation are determined for the bar and disk of the LMC. The ages of the stellar populations in the bar differ from that for the galaxy as a whole: in the bar, there is a deficit of intermediate-age clusters $\left(5 \times 10^{8}-6 \times 10^{9} \mathrm{yr}\right)$ and an excess of young clusters with ages $3 \times 10^{7}-5 \times 10^{8} \mathrm{yr}$. This indicates that the star formation history in the bar differs from that in regions outside the bar at the same distances from the center of the galaxy. Data on the composition of the old stellar populations in the bar and the disk of the LMC and the spatial distribution of intermediate-age clusters in the galaxy suggest that the LMC bar formed $(2-6) \times 10^{9}$ years ago. Deviation of colors of bars from the normal color sequence of galaxies on several two-color diagrams is found using multicolor surface photometry for eight barred galaxies. Using results of evolutionary synthesis modeling, I can model the abnormal colors of bars by an intermediate-age star $\left(\sim 10^{9} \mathrm{yr}\right)$ deficiency. I find that the deficiency of an intermediate-age stellar population is a general property of bars.
\end{abstract}

There are many studies of multi-color photometry of both early-type and late-type barred galaxies in which deviations of the color indices of bars from the normal color sequence for galaxies are noted in the two-color diagrams. These deviations increase toward the ends of the bars. This can be explained by a deficit of intermediate-age stars (or an excess of old and young stars) in the bars of these galaxies (Esipov et al. 1993). The LMC is a unique example of a barred galaxy in which we can estimate differences between the stellar populations in the bar and disk by directly comparing the properties of clusters with known ages in the bar and in the galaxy as a whole.

The age distribution of clusters of our sample in the LMC bar differs from one in the LMC disk. Figure 1a shows distributions of the number of clusters per unit age interval $(d N / d t)$ in the bar and disk of the LMC for our sample and the data of Bica et al. $(1992,1996)$. This distribution characterizes and compares the star formation history in the bar and the galaxy as a whole. As one can see in Figure 1a, there is a deficit of old and young clusters and an excess of clusters with ages $3 \times 10^{7}-5 \times 10^{8} \mathrm{yr}$ and of very young clusters with ages less than $2 \times 10^{6} \mathrm{yr}$ in the LMC bar. Evidently the star formation history 

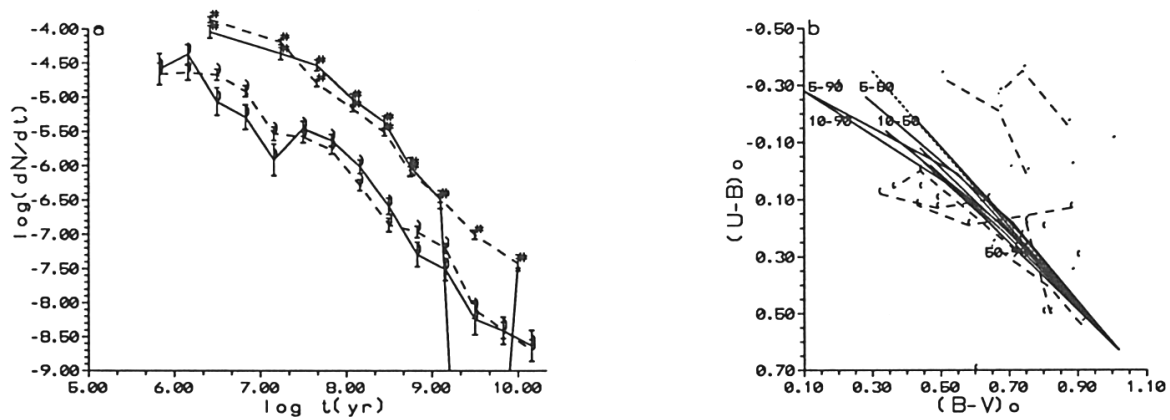

Figure 1. (a) Distribution of the number of clusters per unit age interval in the bar (solid curve) and the disk (dashed curve) of the LMC as a function of age, based on our data (asterisks) and the data of Bica et al. 1992, 1996 (squares). Poisson errors are indicated. All data are normalized. The curves for the data of Bica et al. are shifted upward by $\log (d N / d t)=1.0$. (b) Model sequences (solid lines) for systems with no intermediate-age stars (a range of ages is marked by numbers in Gyr) for the $(U-B)_{0}-(B-V)_{0}$ two-color diagram. The short dashed lines are the normal color sequence of galaxies. The observed colors for galaxy bars (asterisks) are indicated. The dashed curves show how color indices change along semi-major axis of bars.

in the bar differs from that for the disk. This is true for regions in and outside the bar at the same distances from the LMC center.

I studied the color indices in eight early- and late-type barred galaxies along the bar major axis, and also the general color indices for 67 barred galaxies. The bar color indices corrected for Galactic and inner absorption are shown in the two-color diagram $(U-B)_{0}-(B-V)_{0}$ in Figure 1b. In this figure, the color indices of bars clearly separate from the normal color sequence (NCS) of galaxies.

Using a modified PEGASE program, I studied the color indices of the stellar system with no intermediate-age star formation $\left(5 \times 10^{8}-9 \times 10^{9} \mathrm{yr}\right)$ using evolutionary synthesis modeling. Stellar systems with different SFR(t) were examined and the results are presented in Figure 1b. This figure shows that a deficiency of intermediate-ages stellar population (with ages $(1-9) \times 10^{9}$ years) is observed in bars. The same result is seen in other two-color diagrams.

These results show that a deficiency of intermediate-age stellar populations is a general property of bars.

Acknowledgments. I would like to thank A.V. Zasov, Yu.N. Efremov, and V.V. Kravtsov for advice and comments. I am most grateful to the organizers of the Symposium for financial support.

\section{References}

Bica, E., Claria, J.J., \& Dottori, H. 1992, AJ, 1992, 103, 1859

Bica, E., et al. 1996, A\&AS, 102, 57

Esipov, V.F., Zasov, A.V., \& Popravko, G.V. 1993, AZh, 70, 1 